\title{
Distinctive Image Region Features from Color Invariant Moments
}

\author{
L. Guo ${ }^{1,2,3}$, Z. Shi ${ }^{2}$, J. Zhao ${ }^{1}$ and R. Zhang ${ }^{1}$ \\ 1.Faculty of Information Science \& Engineering, \\ Ningbo University \\ No.818, Fenghua Road, Ningbo City, \\ Zhejiang, China \\ guolijun@nbu.edu.cn \\ zhao_jieyu@nbu.edu.cn \\ zhangrong@nbu.edu.cn
}

2.Institute of Computer Technology, CAS

Beijing, China

shizp@ics.ict.ac.cn

3.Graduate University of Chinese Academy of Sciences

Beijing, China

\begin{abstract}
This paper proposes color invariant moment features based on color clustering. Similarity measure method based on the color invariant moment descriptors is given. This method is applied in a practical application system. The experimental result shows that, the proposed feature has both a wider and steadier invariant and a stronger ability in color based image region similarity discriminating than the traditional color features.
\end{abstract}




\section{Introduction}

Some problems of image processing can be dealt with finally by computing similarity of two image regions, for example, CBIR or object class recognition[1][2]. A number of features, such as color, texture, shape, corner, et al, can be used for computing similarity of two image regions [3]. As such, some descriptors invariants based on simple features can also work well like shape context, differential invariants, SIFT and moment invariants [4]. But in some practical applications, for example, for judging if two regions from two images are of the same object, especially when the detected object is non-rigid (e.g., human body), it is not applicable of these features and descriptors. The fact is that it may be difficult even impossible to get satisfying results only by them. Factually, the perplexing problem arises in comparison and discrimination of human target from different monitor videos [5] [6], in which the images often are of low resolution and from different types of video equipment, and there exist changes in viewpoint, size, background, illustration and body deformation. In this case, the clothing color is a more reliable feature than other image content (assumed that the detected human does not change his/her dress). However, the previous experiments have proved that the traditional color features are inefficient in the actual applications. This paper proposes the introduction of color invariant moment features based on color clustering information. The experimental result shows that, the proposed feature has not only a wider and steadier invariance but also a stronger ability in color based image region similarity discriminating than the traditional color features.

The reminder of this paper is organized as follows. First, color invariant feature and relative issues are discussed in Section 2; in Section 3, the presented color invariant moment feature and the methods of similarity measurement are described in detail; and in Section 4, the experimental results on discriminating similarity of two image regions based on clothing color are given; followed by the conclusion and future work in Section 5.

\section{Color invariant feature}

Color features have been applied wildly by virtue of relative low dependence on location, size and direction; and good robustness as well as being easy to extract. Generally, color features include color histogram, color moments, color correlogram, et al. Though color histogram are based on various color spaces, HSV color space and CIE Lab color space are more often used in practice because their space structures are in accordance with people's subjective judgment much better [7][8]. Gevers[9] adopts a transform $C 4=(R-G) /(R+G), C 5=(R-B) /(R+B)$, $\mathrm{C} 6=(\mathrm{B}-\mathrm{G}) /(\mathrm{B}+\mathrm{G})$, to realize the transformation from $\mathrm{RGB}$ space into $\mathrm{C} 4 \mathrm{C} 5 \mathrm{C} 6$ space, in which color component $\mathrm{Ci}$ does not vary with the angle and intensity of 
the incident light and other, and as a result, a invariant histogram is obtained. Furthermore, color histogram based similarity can be computed with L1 distance and L2 distance based on corresponding vector spaces and histogram intersection which is more often used in practice. However, results, using color histogram based similarity measurement, are unsatisfactory because of the problem of color quantization which probably gives rise to very small similarity values among almost the same images. Color moments, based on the assumption that the distribution of color in an image can be interpreted as a probability distribution, are more simple and effective measure presented by Stricker and Orengo[10]. Probability distributions are characterized by a number of unique moments. Consequently, the distribution of color in an image can be represented as three central moments that are means, variance and skewness. Compared with color histogram, this method avoids feature quantization. But it is often used before other features to help narrow range as a filter due to the limited discrimination ability of color moments. Totally, traditional color features, generally used with other features, can describe global color distributions of selected regions in an image but fail to describe accurate color distributions. Conclusively, traditional color features are unable to distinguish if two regions are from the same object.

The traditional moment invariants are computed for an image region based both on the pixels on the shape boundary and the interior. Hu [11] derived functions based on the scale normalized central moments, and proposed seven RST (Rotation, Scaling and Translation) invariants of the second and third-order moments. Mindru et al. [12] proposed several types of generalized color moment invariants based on the $\mathrm{Hu}$ group methods. These invariants are developed according to the combinations of geometric and photometric transformations. Obviously these generalized color moments aren't fit in the application proposed above because it is difficult to obtain geometric features and the objects are nonrigid.

Geusebroek [13] obtained 6 kinds of color invariants in their physics experiment. These color invariants can be used to extract correct color distribution features of image regions. But these features are obtained in different physical environment which not only include factors such as illumination and viewpoint, but also include material quality (reflection coefficient), roughness degree that we cannot consider in common applications.

In order to avoid the deficiencies of color features, considering changes in viewpoint and illumination, we present a novel color feature based on color invariant moments. The basis of the feature lay in color cluster. The experimental results have shown this method works better than other color invariant features in similarity distinguishing of two image regions under the condition of fixed background and single camera. 


\section{Color invariant moments}

Different from common image retrieval, this paper presents a similarity calculation method based on color invariant moment.The method is described in detail as follows.

\subsection{Color clustering and color invariant moments}

The proposed color invariant moments are founded on color clustering. So the preliminary work is color clustering calculation on object image regions. K-mean clustering method is applied. For convenience, object image mentioned below refers to object image region and retrieved image refers to retrieved image region.

In the process of clustering, means and variances corresponding to color channels of each clustering color are calculated, which are respectively represented as means $[\mathrm{k}][\mathrm{ch}]$ and vars $[\mathrm{k}][\mathrm{ch}]$ ( $\mathrm{k}$ is the clustering color index, ch is the color channel index), and pixel number of each clustering color, represented as $\operatorname{pck}(\mathrm{k}$ is the clustering color index), is calculated too. In general case, the clustering color number is specified artificially according to object image.

After color clustering, color variant moments for object image are computed according to the following formulas:

Formula 1:

Xmoment $[k]=\sum_{i=0}^{I . h t e i g h t} \sum_{j=0}^{I . \text { width }} i, \quad k \in\left[\begin{array}{lll}1 & . . & N\end{array}\right] \quad \operatorname{Color}\left(p_{i j}\right)=k$

Formula 2:

Ymoment $[k]=\sum_{i=0}^{I . h \text { teight }} \sum_{j=0}^{I . \text { width }} j, \quad k \in\left[\begin{array}{lll}1 & . . & N\end{array}\right] \quad \operatorname{Color}\left(p_{i j}\right)=k$

Where Pij represents the image pixel at the ith row and the jth column in an object image and color $(\mathrm{Pij})=\mathrm{k}$ further shows that the color of the current pixel belongs to the k-th color cluster. Xmoment[k], Ymoment[k] and pck construct moment of the k-th color cluster. In other words, color invariant moments include 3 channels. 


\subsection{Color projection}

Obtaining color invariant moments of retrieved image is divided into two steps, color projection and feature computation. Color projection is a projection from pixels in retrieved image region onto cluster colors in object image. In this process, the pixels in retrieved image region are compared with the values of means and variances of each color in object image obtained by color clustering so as to distinguish the cluster class the retrieved image region belongs to. Formally this is described as :

Formula 3:

$\sum_{k=1}^{N 1} \sum_{c h=1}^{N 2}\left|p_{i j}[c h]-\operatorname{means}[k][c h]\right|>2 * \sqrt{\operatorname{var} s[k][c h]}$

Formula 4:

$\sum_{k=1}^{N 1} \frac{\sum_{c h=1}^{N 2}\left(P_{i j}[c h] * \text { means }[k][c h]\right)}{\sqrt{\sum_{c h=1}^{N 2}\left(p_{i j}[c h] * p_{i j}[c h]\right)} * \sqrt{\sum_{c h=1}^{N 2}(\operatorname{means}[k][c h] * \text { means }[k][c h])}}>$ const

Where $\mathrm{k}$ is the index of cluster color, ch is the current color channel index, N1 and N2 respectively represent the number of clustered colors and the number of color channels, Pij[ch] is the pixel value at the $\mathrm{i}$-th row and the $\mathrm{j}$-th column in reretrieved image, means[k][ch] and vars[k][ch] respectively represent mean and variance of the ch color channel of the k-th color, which are obtained from object image clustering mentioned above. If a pixel in retrieved image simultaneously satisfies Formula 3 and Formula 4, it can be determined that the color of the pixel is the same to the $\mathrm{k}$-th clustered color in object image and the pixel value is assigned as the corresponding cluster color index and otherwise, it is assigned as 0 . After projection of retrieved image (single component/channel image is acquired), color cluster information of retrieved image is acquired and therefore color invariant moment features are computed by Formula 1 and Formula 2. 


\subsection{Color similarity computing}

After obtaining color variant moments of the object image and the retrieved image, in order to compute color similarity of the two images we adopt the following formulas, where Ixmo [k] and Iymo [k] (Sxmo [k] and Symo [k]) represent the moment of the kth clustering color of object image (of retrieved image); Ipck (Spck) represents the number of pixels belonging to the kth clustering color in object image (in retrieved image). Finally, color similarity is calculated by Formula 8, where W1, W2, W3 are weight parameters, which are determined from experience.

Formula5:

$$
S_{p c}=\frac{\sum_{k=1}^{N 1}\left(I p c_{k} * S p c_{k}\right)}{\sqrt{\sum_{k=1}^{N 1}\left(I p c_{k} * I p c_{k}\right)} * \sqrt{\sum_{k=1}^{N 1}\left(S p c_{k} * S p c_{k}\right)}}
$$

Formula 6:

$$
S_{x}=\frac{\sum_{k=1}^{N 1}(\operatorname{Ixmo}[k] * \operatorname{Sxmo}[k])}{\sqrt{\sum_{k=1}^{N 1}(\operatorname{Ixmo}[k] * \operatorname{Ixmo}[k])} * \sqrt{\sum_{k=1}^{N 1}(\operatorname{Sxmo}[k] * \operatorname{Sxmo}[k])}}
$$

Formula 7:

$$
S_{y}=\frac{\sum_{k=1}^{N 1}(\operatorname{Iymo}[k] * \operatorname{Symo}[k])}{\sqrt{\sum_{k=1}^{N 1}(\operatorname{Iymo}[k] * \operatorname{Iymo}[k])} * \sqrt{\sum_{k=1}^{N 1}(\operatorname{Symo}[k] * \operatorname{Symo}[k])}}
$$

Formula 8:

$$
S=W_{1} * S_{p c}+W_{2} * S_{x}+W_{3} * S_{y}\left(W_{1}+W_{2}+W_{3}=1\right)
$$


Color invariant moments and color similarity computed by the above formulas have the following strong features: (1) Extraction of color invariant moments does not involve color space quantization; (2) Based on color clustering, this method is able to eliminate the difference of color systems caused by different devices; (3) Location information and number information of pixels are taken into account in the calculation of color invariant moment and color similarity so as to successfully eliminate the effect of viewpoint and illumination. In the next section we will give experimental results which can prove them.

\section{Results}

We have applied the proposed color invariant moment features to the practical human detection system based on clothing. Considering that the detected object is a region in an image, we design two experiments, shown in Figure 1 and Figure 2 respectively.

In Figure 1, there is one object image and four retrieved images. All retrieved images, which are different in background, viewpoint and illumination and are from different image devices, and the object image include the same human object. HSV space based color histogram features, C4C5C6 space based color histogram features and color invariant moments are applied separately. The experimental results show that the novel features work better than HSV color histogram features and C4C5C6 color histogram features.

In this experiment, by transforming RGB space of the original image into the corresponding HSV space and C4C5C6 space we obtain HSV color histogram features and $\mathrm{C} 4 \mathrm{C} 5 \mathrm{C} 6$ color histogram features. Following that, by OpenCV based histogram computation and comparison similarity measure values are obtained. Relevant parameters in color invariant moment features are set as follows: the number of cluster color $\mathrm{N} 1=2 ; \mathrm{W} 1=0.6 ; \mathrm{W} 2=\mathrm{W} 3=0.2$; in formula 4 , const $=0.95$. The next experiment applies the same parameters.

Figure 2 illuminates another experiment. The experimental results show that bigger is the value of Formula 8 , the similarity of two image regions is higher. Meanwhile, the experiment results support the conclusion drawn from previous experiment. 


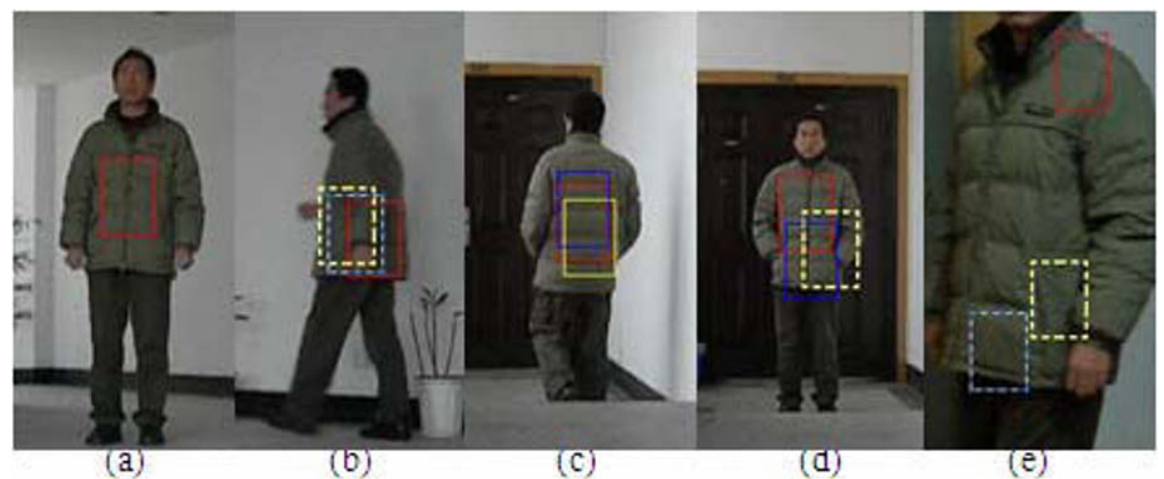

Fig.1 . (a) is the object image with specified object region in red. (b), (c), (d) and (e) are retrieved images (obtained from video by moving object detection), in which detected results based on HSV color histogram features, C4C5C6 color histogram features and color invariant moments are shown respectively in yellow, blue and red. In (b), the similarity measure values are 0.172 , 0.260 and 0.972 corresponding to the three feature methods; in (c), $0.797,0.882$ and 0.987 ; in (d) $0.425,0.760$ and 0.998 ; in (e), 0.423、0.566、0.981.

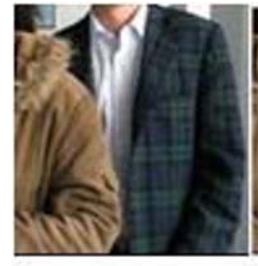

(a)

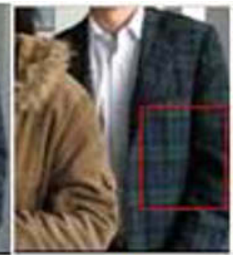

(b)

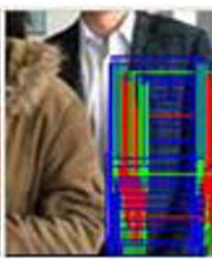

(c)

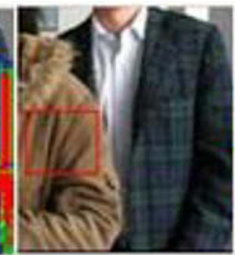

(d)

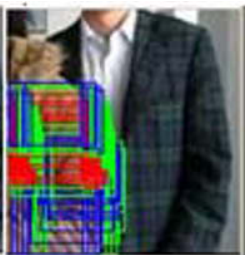

(e)

Fig.2 . (a) is the original image. (b) and(d) show object color region in red. (c) and(e) are retrieval results corresponding to the object regions in (b) and (d). Regions with higher similarity in retrieved images are outlined in red, followed by green (threshold $>0.98$ ) and blue (threshold $>0.97)$

\section{Conclusion}

In this paper, we present color invariant descriptors. Analysis on experimental data show that the descriptors have some shortcomings: (1) Mistaken detection probably happens as other descriptors but it can be reduced by adding texture descriptor. This is our prime work in future. (2) The color invariant descriptors proposed in this paper are not suitable for images which include a great number of colors in detected regions. Because more colors, color cluster error is bigger and the discrimination ability of the color invariant moment descriptors based on color cluster gets worse. 
Our future work includes two aspects. On one hand, we will revise and optimize the color invariant moment descriptors to improve the discriminating ability and expend the application range. On the other hand, aiming at improving detection efficiency, we will perfect the object human detection framework by improving color cluster method and adding moving human detection, texture feature into the system.

\section{Reference}

[1] Gevers, T., Smeulders, A.W.M., PicToSeek: Combining Color and Shape Invariant Features for Image Retrieval,IP(9), No. 1, January 2000, pp. 102-119.

[2] Gy. Dorko and C. Schmid. Object class recognition using discriminative local features. IEEE Transactions on Pattern Analysis and Machine Intelligence, 2004. submitted.

[3] FAN Zi-zhu ,A Survey of Content-based Image Retrieval ,Journal of East China Jiaotong University .

[4] K. Mikolajczyk, C. Schmid, A performance evaluation of local descriptors, in: Proceedings of IEEE Conference on Computer Vision and Pattern Recognition, Madison, USA, June 2003.

[5] J. Sivic, F. Schaffalitzky, A. Zisserman, Efficient object retrieval from videos, in: Proceedings of the 12th European Signal Processing Conference, Vienna, Austria, 2004.

[6] Mark. S. Drew, Jie Wei, Ze-Nian Li, On Illumination Invariance in Color Object Recognition, Technical report 1997, School of Computing Science, Simon Fraser University, Vancouver, Canada.

[7] DOU Jian-jun, WEN Jun, LIU Chong-qing. Histogram-based color image retrieval. Infrared and Laser Engineering, 1999(1)

[8] Yao Qiong, Lai Jianhuang, Feng Guocan. Color-based Image Retrieval :An Overview of Current Research, Journal of Image and Graphics. 2003(z1)

[9] Gevers, T., Smeulders, A.W.M., PicToSeek: Combining Color and Shape Invariant Features for Image Retrieval,IP(9), No. 1, January 2000, pp. 102-119.

[10] M. Stricker, M. Orengo, Similarity of color images, in: Proc. SPIE Storage and Retrieval for Image and Video Databases, San Jose, 1995, pp. 381-392.

[11] M. Hu, Visual pattern recognition by moment invariants, IEEE Transactions on Information Theory IT-8 (1962) $179-187$.

[12] F. Mindru, T. Moons, L.V. Gool, Color-based moment invariants for the viewpoint and illumination independent recognition of planar color patterns, in: Proceedings of International Conference on Advances in Pattern Recognition, 1998.

[13] J. M. Geusebroek, R. van den Boomgaard, A. W. M. Smeulders, and H. Geerts. Color invariance. IEEE Trans. Pattern Anal. Machine Intell., 23(12):1338-1350, 2001. 